\title{
On-Tree Detection and Counting of Mature and Immature Fruit of Carica Papaya using Image Processing Technique
}

\author{
Prabira Kumar Sethy \\ SUIIT,Sambalpur University \\ Jyoti Vihar, Burla \\ Odisha, India
}

\author{
Shwetapadma Panda \\ SUIIT,Sambalpur University \\ Jyoti Vihar, Burla \\ Odisha, India
}

\author{
Nilamani Bhoi \\ Veer Surendra Sai \\ University of Technology \\ Siddhi Vihar, Burla, \\ Odisha India
}

\begin{abstract}
Papaya (Carica papaya) is a tropical fruit having profitable importance because of its high nutritive and medicinal value. India leads the world in papaya production with an annual yield of about 3 million tons [1]. Before collection of these fruits are graded according to their maturity. The major parameter which is differentiating between mature and immature fruit is its color. And also by counting individually mature and immature fruit which implies knowledge about the productivity of the plant. Image analysis is a technique to count the number of fruits on-Tree. In this paper, we present a method for detecting and counting mature and immature fruits from images taken with a tree. We have demonstrated that the proposed method is able to achieve an accuracy of $89.18 \%$ and $100 \%$ for detecting as well as counting of immature and mature fruit respectively.
\end{abstract}

\section{Keywords}

Fruit counting; Carica Papaya; Randomized Hough Transform; Texture Analysis; K-means Clustering; Erosion \& Dilation

\section{INTRODUCTION}

Papaya is one of the leading fruit crops cultivated in tropical and subtropical zones [2]. It was rapidly spread to other parts of the world due to its importance and popularity. Papaya is a fast - growing, semi-woody tropical herb. The fruit of Papaya is melon-like oval to nearly round, somewhat performs or elongated club-shaped. $15-50 \mathrm{~cm}$ long and $10-20 \mathrm{~cm}$ thick and weighing up to approximately $9 \mathrm{~kg}$ (Morton 1987), semi-wild (naturalized) plants bear small fruits $2.5-15 \mathrm{~cm}$ in length [2]. The skin is waxy and thin but fairly tough. When the fruit is immature, it is rich in white latex and the skin is green and hard [2]. As ripening progress, papaya fruits develop a light or deep- yellow-orange colored skin while the thick wall of luscious flesh becomes fragrant having yellow-orange or various shades of salmon or red [2]. There are many numbers of applications for detecting and counting fruits from images or videos are already exists. Though numerous research papers have been published to detect and count fruits on-tree, still the need for developing a more sophisticated technique is that for more complex images where there is a low contrast between fruits and other parts (leaves, petiole, leaf axils, node, internodes, and flowers), foreground occlusions and cluttered backgrounds [3].There is much application for identifying fruits e.g. identifying red apples in green canopies [4]. For measuring the size and counting apple fruits thermal imaging application was used [5]. In this work, they discriminate the round apple from the leaves by using morphological operation and shape constraint. Jimenez, Jain, Ceres, and Pons (1999) provided a review of many vision systems to identify fruits for automated harvesting using a laser range-finder [6]. Zhao, Tow, and Katupitiya (2005) presented methods to recognize apples grown on plants, where they used the texture and redness color [7]. It was shown that redness works equally well for green apples as for red ones. Yang, Dickinson, Wu, and Lang (2007) proposed a technique to identify red tomato and detect cluster positions for harvesting application of tomato [8]. Kitamura and Oka (2005) described a robot which recognizes and pick-up sweet peppers in greenhouses, but it only works under fixed lighting conditions [9].Chinchuluun and Lee (2006) developed a fruit identification method using the Watershed transform to segment and split overlapping fruit for accurate fruit counts and estimate the result [10].All these studies are to detect Orange-colored fruit from the green-colored leaf. Kane and Lee (2006) use a spectrophotometer to detect green fruits from leaves [11]. The overall goal of this study was to develop a prototype for detecting green fruit in papaya trees. In most agricultural machine vision practice, a red, green, and blue (RGB) color camera is used. However, it is difficult to retrieve green papaya fruit from background leaves using these images because the leaves and the fruits are alike. During ripening procedure, the color of papaya fruit varies from mature green to Pale yellow color. The Ripeness of a papaya can be estimated through its surface color. The surface color of a papaya is a major factor in determining its ripeness. On this basis, we proposed a color-based algorithm for recognizing papaya. The proposed algorithm has the capability to recognize papaya individually. Recognizing papaya from the background is successfully done by using morphologically open Binary operation. However, the mature papaya from immature ones can be detected by K-means clustering operation.

\section{MAJOR CHALLENGES}

- The color of the papaya fruits and leaves are similar i.e. deep Green. So it is difficult to segregate fruits from the foreground.

- Errors free Counting of individual fruits from a bunch of fruit even if they are in an overlapping manner.

- The shape of papaya fruit and peak portion of the leaf is similar i.e. elliptical or oval in shape. So it's difficult to detect the fruit from leaves.

- Separately counting the mature and immature papaya fruits.

In this paper, the ability of an image processing technique is based on color based segmentation using K-means clustering and has evaluated for recognizing Pale yellow papaya. 
Additionally, the erosion and dilation method with Randomized Hough Transform method is introduced to separate touching or overlapping papayas. Research based on papaya fruit is very useful for post harvesting in the agricultural field. The fruits are packed separately and send to market for commercial purpose. Mature papaya can be used for skin care, hair revitalizes, health benefits, control dandruff, promotes cardiovascular health, prevent macular degeneration, aids digestion, reduce inflammation in lungs. Immature papaya can be used for health benefits and for the vegetable purpose.

\section{METHODOLOGY}

The fruits /vegetables counting algorithm are a sequence of stages in that the initial stage has received raw data in an organized form. However, occlusion, varying illumination, and similarity with the background make fruit identification a very challenging task. Efficient detection of fruits and vegetables in their natural environment and computing a reasonable prediction of the number of fruits and vegetables are examples of the main applications in agriculture.

\subsection{Input Image}

At fast the image is taken by the conventional RGB camera. Then the image, which was taken by the user in a healthy weather at normal temperature is read by the software (FIG.1a).

\subsection{Texture Analysis}

This method is choosing to define the edge of each of the papaya fruit in the image. It also quantifies the qualities of the particular image such as rough, smooth, silky or bumpy (FIG.1 -b).

\subsection{Pre-Processing Of the Image}

\subsubsection{Sharpness of image}

To sharpen the image, the image will undergo Gaussian Blurring filter. Here the default threshold value i.e. 0.8 (FIG.1- c).

\subsubsection{Binary image}

To remove the small patches of unwanted pixel area, the image is converting to binary form. (FIG.1-d).

\subsection{Morphological Open Binary Image}

It is used to remove small objects from a particular image. STREL function used to create morphological structuring element (FIG.1-e).

STREL: Flat structure of neighbor element having neighborhood 8.Here we use eye (n) function to return n-by-n identity matrix.

\subsection{Erosion Operation}

To remove noise and defined the shape of the papaya fruit, erosion operation is used (FIG.1-f).
$B W 2=$ imerode $($ bwAreaOpen $B W, S E)$

\subsection{Combine Erosion and Dilation Operation}

The range of upper bound and lower bound are $\mathrm{LB}=60000$ and $\mathrm{UB}=460$.To keep only objects between 60000 pixel and 460 pixels in area. The function "BWareaopen" is used to remove the objects which have an area greater and lesser than some threshold area and extracting only the objects which have the area in between them (FIG.1-g).

\subsection{Detection of Binary Region}

The binary edge of image is computed using edge function. Then convert the matrix to a grayscale image. Here the image shows the region of the potential area of the papaya fruit. The area of each of the region is then scanned. The region that might represent the papaya fruit is detected (FIG.1-h).

\section{FIGURES}
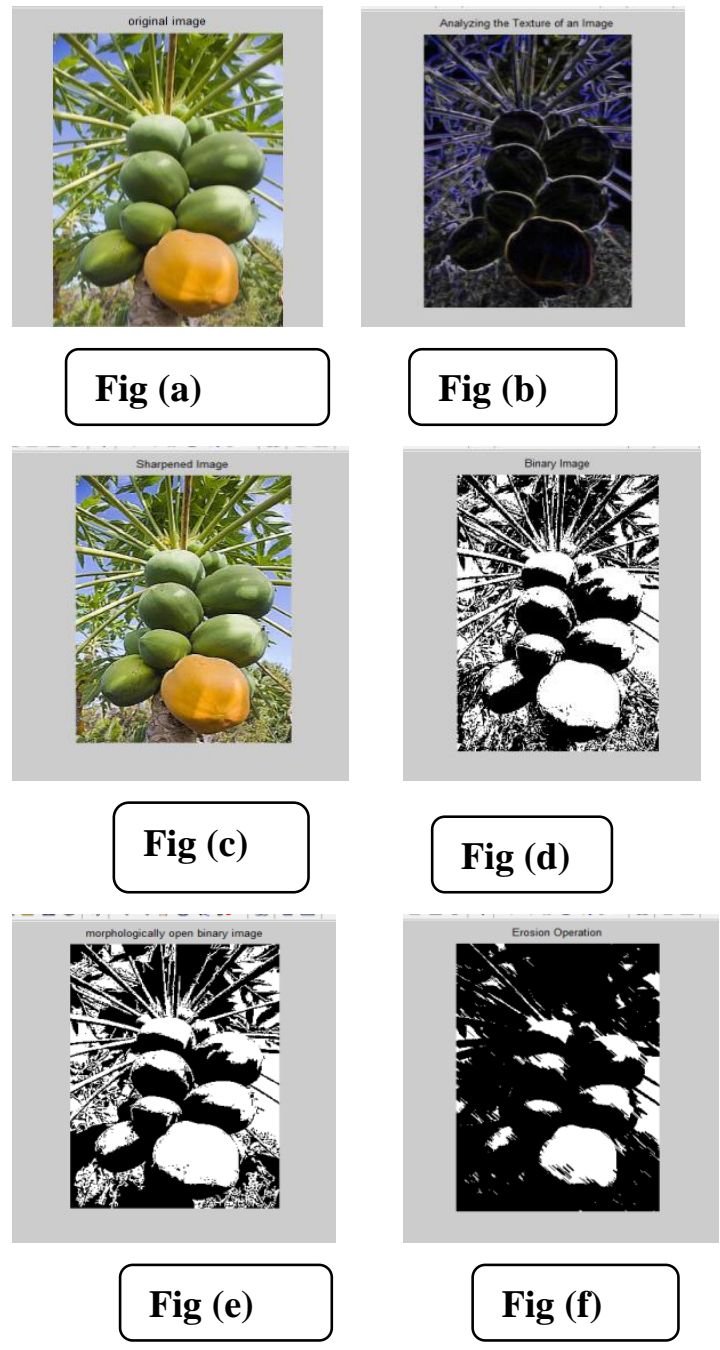

Erosion operation: 

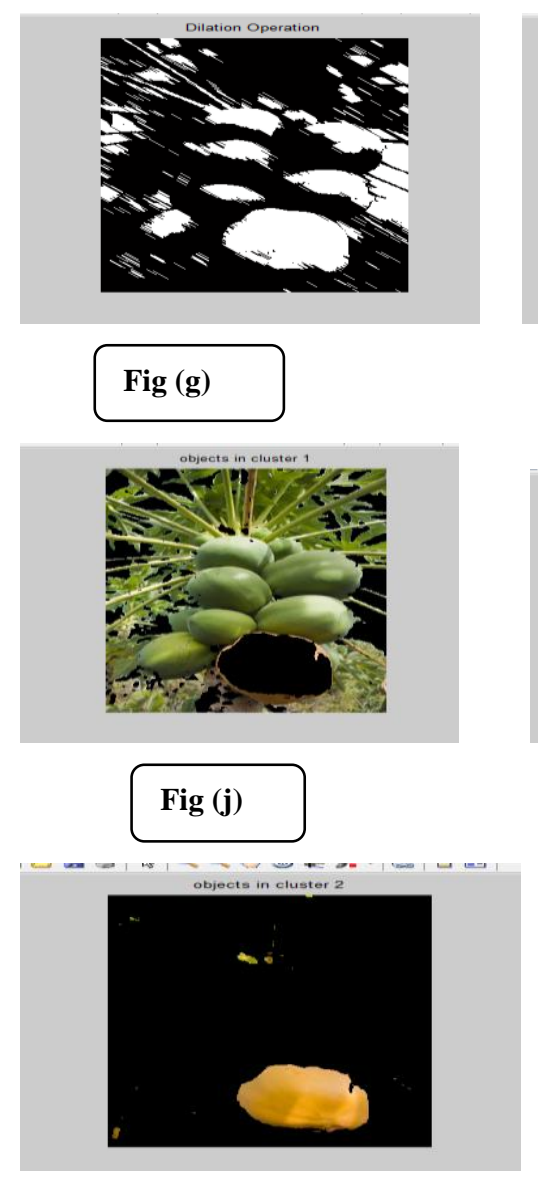

Fig (m)

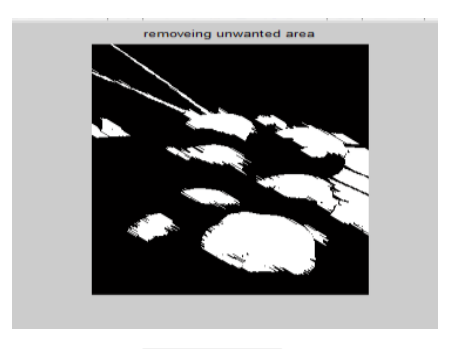

Fig (h)
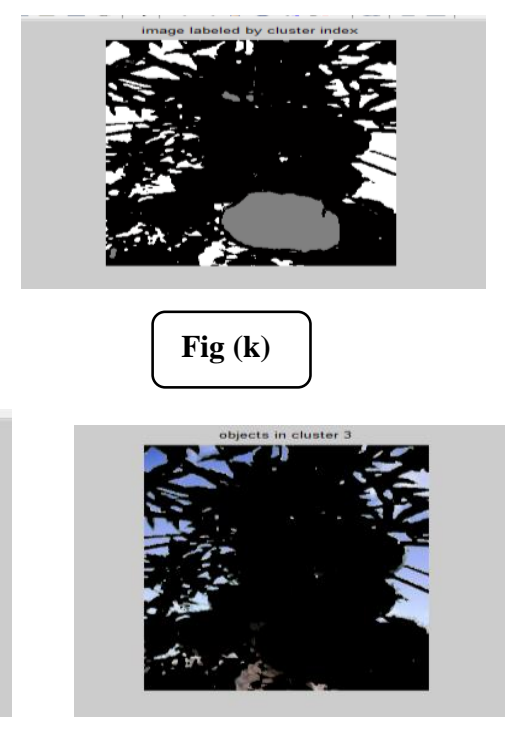

Fig (n)

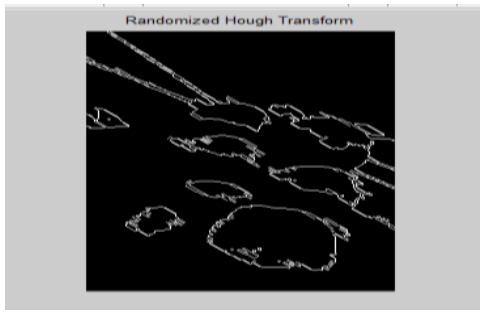

Fig (i)

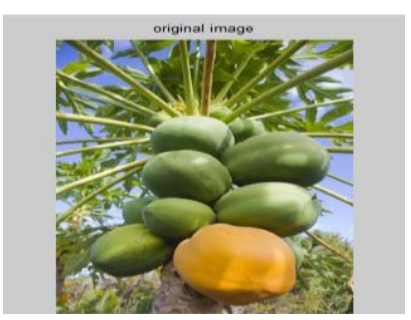

Fig (l)

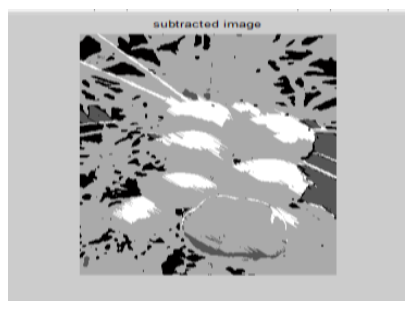

Fig (o)

FIG.1Image processing operation for Detection and counting operation of mature and Immature papaya fruits (a)Original image (b)Analyzing the texture of an image (c)Sharpened Image (d)Binary image (e)Morphologically open binary image (f)Erosion operation (g)Dilation operation (h)Removing unwanted area (i)Randomized Hough Transform (j)Original image (k)Image labeled by clustering index (l)Object in cluster1 $(\mathrm{m})$ Object in cluster 2 (n)Object in cluster 3 (o)Subtracted image.

\subsection{Detection of Ellipse Using Randomized Hough Transform}

The basic Hough transform can be used to detect lines and curves in an image also used for circle detection. Here the problem is that it is failure to detect specific curves. Having low accuracy is the major problem if the accumulator is not properly defined. So Randomized Hough transform (RHT) is choosing to detect the ellipse (FIG.1-i). The operation is as follows.

$$
A(x-p)^{2}+2 B(x-p)(y-q)+C(y-q)^{2}=1
$$

$\mathrm{P}=\mathrm{x}$ coordinate of ellipse centre.

$$
\mathrm{q}=\mathrm{y} \text { coordinate of ellipse centre }
$$

$\mathrm{a}=$ semi-major axis length

$\mathrm{b}=$ semi-major axis length

Here the information needed is the sub axis of the $\mathrm{x}$-axis of the non-tilt ellipse, sub axis of the y-axis of the non-tilt ellipse orientation in a radius of the ellipse (tilt), center at the $\mathrm{x}$-axis of tilt ellipse and center of the y-axis of the tilted ellipse. Then the area of the ellipse is compared to the area of the binary region that occupied in the ellipse shape.

\subsection{Counting}

It uses label connected components in the binary image.

\section{$[L, n u m]=\operatorname{bwlabel}(B W, n)$}

Here it returns in "num" the number of connected objects found in BW.

\section{DETECTION OF MATURE FRUIT}

It totally based on color-based segmentation using K-means clustering .The clustering process is repeat 3 times to avoid local minima. Then by using the counting method we count the number of mature fruit among all the fruit in that image (FIG.1 (j-n).

\subsection{Detection of Immature Fruit}

The subtraction operation is use to detect the immature fruit. Then again use the counting method to count the number of immature fruit. (FIG.1 (0)). 


\section{FLOWCHART}

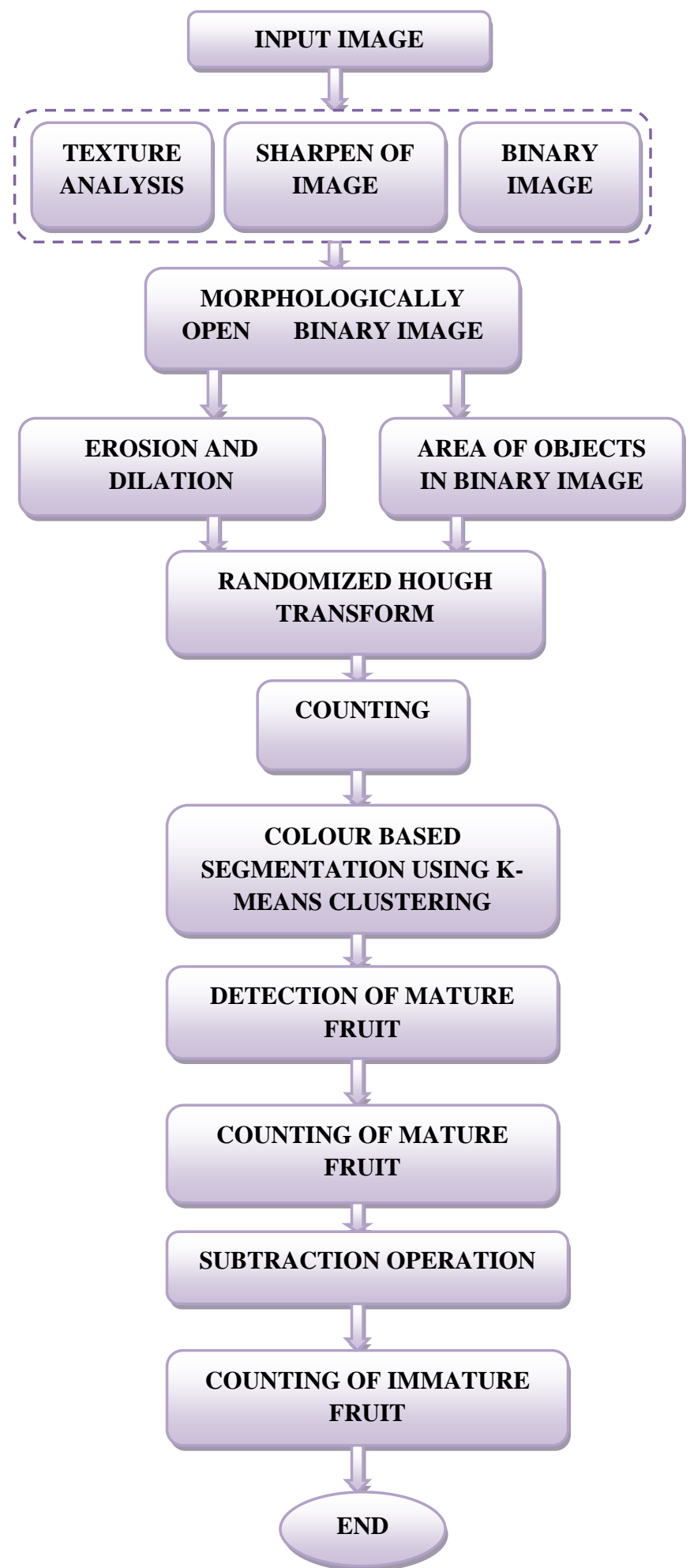

\section{RESULTS AND DISCUSSIONS}

The proposed method can detect and count both mature and immature papaya fruit separately under natural condition. In demostration, 5 number of sample are taken.i.e. FIG.2. $(\mathrm{a}-\mathrm{e})$. The demonstration result for both immature and mature fruit counting is shown in Table-1and Table- 2 respectively. 

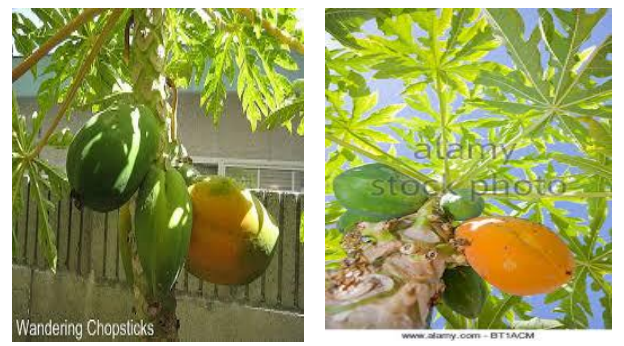

Fig (b)

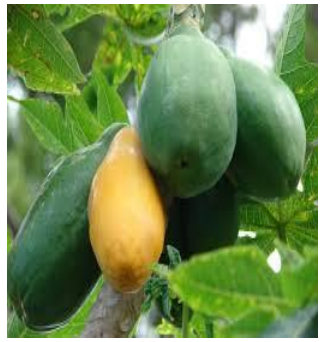

Fig (c)

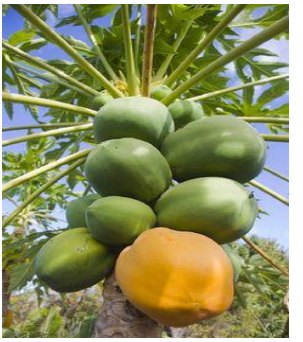

Fig (d)

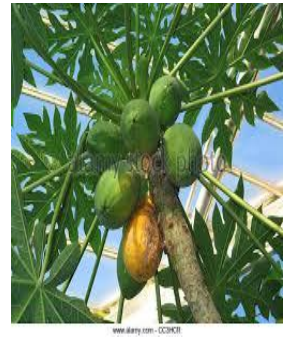

Fig (e)

FIG .2 Samples of images is taken to know the percentage of accuracy in counting for both mature and immature papaya fruit.

\begin{tabular}{|c|c|c|c|c|c|c|c|c|c|}
\hline \multicolumn{5}{|c|}{$\begin{array}{l}\text { Table } 1 \text { Result of Immature Carica Papaya fruit Detection and } \\
\text { Counting of five samples of the image. }\end{array}$} & \multicolumn{5}{|c|}{$\begin{array}{c}\text { Table } 2 \text { Result of Mature Carica Papaya fruit Detection an } \\
\text { Counting of five samples image. }\end{array}$} \\
\hline $\begin{array}{l}\text { Sl. } \\
\text { No. }\end{array}$ & $\begin{array}{c}\text { No. of } \\
\text { fruits } \\
\text { count } \\
\text { manually }\end{array}$ & $\begin{array}{c}\text { No. of } \\
\text { fruits } \\
\text { count by } \\
\text { proposed } \\
\text { method }\end{array}$ & $\begin{array}{c}\text { Percentage } \\
\text { of } \\
\text { Accuracy }\end{array}$ & $\begin{array}{l}\text { Mean } \\
\text { Value }\end{array}$ & Sl. No. & $\begin{array}{c}\text { No. of } \\
\text { fruits } \\
\text { count } \\
\text { manually }\end{array}$ & $\begin{array}{c}\text { No. of } \\
\text { fruits } \\
\text { count by } \\
\text { proposed } \\
\text { method }\end{array}$ & $\begin{array}{c}\text { Percentage } \\
\text { of } \\
\text { Accuracy }\end{array}$ & $\begin{array}{l}\text { Mean } \\
\text { Value }\end{array}$ \\
\hline 1 & 8 & 7 & $87.5 \%$ & & 1 & 1 & 1 & $100 \%$ & \\
\hline 2 & 2 & 2 & $100 \%$ & & 2 & 1 & 1 & $100 \%$ & \\
\hline 3 & 4 & 3 & $75 \%$ & $89.18 \%$ & 3 & 1 & 1 & $100 \%$ & $100 \%$ \\
\hline 4 & 3 & 3 & $100 \%$ & & 4 & 1 & 1 & $100 \%$ & \\
\hline 5 & 6 & 5 & $83.40 \%$ & & 5 & 1 & 1 & $100 \%$ & \\
\hline
\end{tabular}

\section{CONCLSION}

The proposed method is successfully detecting and counting on-plant mature and immature papaya fruits separately. Basically some work like papaya size grading, sex detection has done but no work has been done for counting of papaya fruit separately for mature and immature one. As basic tools, texture analysis, the sharpness of image, binary image etc are used. The major operation in this process was Randomized Hough Transform was used for detection of elliptical objects; $\mathrm{K}$-means clustering which was used for the detection of mature fruit and subtraction operation was used for detection of immature fruits from the bunch of fruit. In this proposed algorithm the overlapping problem was eradicated by the Randomized Hough Transform as it can separate the papaya individually and by taking the upper bound and lower bound pixel value it can give a result more accurately. Finally the use of morphological binary label connected component for counting of papaya fruit separately for both the cases. By using this proposed method we have got a percentage of accuracy in immature and mature fruit are $89.18 \%$ and $100 \%$ respectively.

\section{FUTURE SCOPE}

This proposed method is still needed to be improving, mainly for immature fruit counting. In this algorithm, one deficiency is that it is required to vary the value of upper bound and lower bound for different sample images. So one can develop such an algorithm, which without variation of the upper bound and lower bound fruit detection and counting may be possible for different sample images.

\section{REFERENCES}

[1] Horticultural statistics at a Glance 2015-Published in India by Oxford University Press, New Delhi,Ministry of Agriculture and farmers Welfare, Government of India 2016.

[2] Jaime A. Teixeira da Silva et al, "Papaya(Carica papaya L.)Biology and biotechnology",Tree and Forestry science and biotechnology @2007 Global science books.

[3] Y. Song et al. "Automatic fruit recognition and counting from multiple images",bios stems engineering 118(2014)203e2 15. 
[4] Bulanon,Kataoka, T., (2002), "A segmentation algorithm for the automatic recognition of Fuji apples at harvest", Biosystems Engineering, 83(4), 405e412.

[5] Stajnko, D., Lakota \& Hocevar, M.(2004), “Estimation of number and diameter of apple fruits in an orchard during the growing season by thermal imaging", Computers and Electronics in Agriculture, 42(1), 31e42.

[6] Jimenez, A., Jain, A., Ceres, R., \& Pons, J. (1999), “Automatic fruit Recognition”, a survey and new results using range/attenuationImages. Pattern Recognition, 32(10), 1719e1736.

[7] Zhao, J., Tow, J., \& Katupitiya, J. (2005), “On- tree fruit recognitionUsing texture properties and color data", In InternationalConference on intelligent robots and systems (pp. 263e268).
[8] Yang, L., Dickinson, J., Wu, Q., \& Lang, S. (2007), “A fruit recognition method for automatic harvesting", In $14^{\text {th }}$ International conference on mechatronics and machine vision inPractice (pp. 152e157)

[9] Kitamura, S., \& Oka, K. (2005), "Recognition and cutting system ofSweet pepper for picking robot in greenhouse horticulture", In IEEEInternational conference on mechatronics and automation (Vol. 4);(pp. 1807e1812).

[10] Chinchuluun and Lee (2006)., "Detection of dropped citrus fruit on the ground and evaluation of decay stages in varying illumination conditions", Computers and Electronics in AgricultureVolume 127, September 2016.

[11] Kane and Lee (2006). "Multispectral Imaging for Infield green citrus identification", Agricultural and biological engineering, university of Florida, Gainesville. 